\title{
2028. Life estimation of the beam with normal distribution parameters and subjected to cyclic load
}

\author{
Changyou Li ${ }^{1}$, Xuchu Wang ${ }^{2}$, Wei Wang 3 , Yimin Zhang ${ }^{4}$, Song Guo ${ }^{5}$ \\ 1,2,3,4 School of Mechanical Engineering and Automation, Northeastern University, Shenyang, China \\ ${ }^{5}$ Information and Control Engineering Faculty, Shenyang Jianzhu University, Shenyang, China \\ ${ }^{1}$ Corresponding author \\ E-mail: ${ }^{1}$ chyli@mail.neu.edu.cn, ${ }^{2}$ wangxuchu102@163.com, ${ }^{3}$ viewii@sina.com, ${ }^{4}$ zhangym@sohu.com, \\ 5guosong@sjzu.edu.cn
}

Received 6 December 2015; received in revised form 30 April 2016; accepted 8 June 2016

DOI http://dx.doi.org/10.21595/jve.2016.16703

\begin{abstract}
This research aims at estimating the life of the beam with normal distribution parameters and subjected to cyclic load. It is tested by Monte-Carlo simulation that the generalized displacement and velocity are normally distributed when the coefficient of variation $(\mathrm{CV})$ of the random parameter is small (generally $C V \leq 0.01$ ). The random perturbation method is employed to estimate the mean and variance of the generalized displacement and velocity. The random dynamic stress and its derivative with respect to the time $t$ of the beam is formulated according to the shape function of beam element and the stress equation in a Euler-Bernoulli beam. Their mean, variance and correlation coefficient are given using the first-order approximation in a Taylor series. Based on Palmgren-Miner rule, the expected cumulative damage equation is given and is used to estimate the life where the random dynamic stress is non-stationary and follows the normal distribution with the nonzero mean at any time $t$. The presented method could also estimate the life of other structure or component which has several normal random parameters, is subjected to cyclic load and obeys the linear dynamics and elastic theory when the random parameter's $C V$ is small.
\end{abstract}

Keywords: life estimation, beam with normal distribution parameters, perturbation approach, non-stationary stochastic stress.

\section{Nomenclature}

$\begin{array}{ll}\mathbf{B} & \text { Random vector composed of all random parameters } \\ \mathbf{B}_{d} & \text { Deterministic part of } \mathbf{B} \\ b & \text { Width of the beam } \\ \mathbf{C} & \text { Damping matrix } \\ C & \text { Material constant in Basquin's relation }\left(N \sigma^{m}=C\right) \\ \mathbf{C}_{d} & \text { Deterministic part of } \mathbf{C} \\ \mathbf{C}_{d, j} & \partial \mathbf{C} /\left.\partial b_{j}\right|_{\mathbf{B}=\mathbf{B}_{d}} \\ C V & \text { Coefficient of variation } \\ D\left(t_{1}, t_{2}\right) & \text { Cumulative damage during the time interval } t_{1} \text { to } t_{2} \\ E & \text { Modulus of elasticity } \\ h & \text { Height of the beam } \\ I(y, \mathbf{B}) & \text { Area moment of inertia of } y \text { cross-section } \\ \mathbf{K} & \text { Stiffness matrix } \\ \mathbf{K}_{d} & \text { Deterministic part of } \mathbf{K} \\ \mathbf{K}_{d, j} & \partial \mathbf{K} /\left.\partial b_{j}\right|_{\mathbf{B}=\mathbf{B}_{d}} \\ L & \text { Total length of the beam } \\ l & \text { Length of the beam element } \\ \mathbf{M}_{\mathbf{M}_{d}} & \text { Mass matrix } \\ \mathbf{M}_{d, j} & \text { Deterministic part of } \mathbf{M}\end{array}$




$\begin{array}{ll}M(y, \mathbf{B}, t) & \text { Bending moment of } y \text { cross-section } \\ n & \text { Total number of the degree of freedom } \\ \mathbf{F}(t) & \text { External load vector } \\ \rho_{1} & \text { Density of the beam } \\ \rho & \text { Correlation coefficient } \\ t & \text { Time } \\ \bar{v}_{\sigma_{z \max }}(t) & \text { Expected number of crossings with positive slope } \\ v(y, \mathbf{B}, t) & \text { Deflection of y cross-section } \\ \ddot{\mathbf{X}} & \text { Generalized acceleration vector } \\ \dot{\mathbf{X}} & \text { Generalized velocity vector } \\ \mathbf{X} & \text { Generalized displacement vector } \\ \ddot{\mathbf{X}}_{d} & \text { Deterministic part of } \ddot{\mathbf{X}} \\ \dot{\mathbf{X}}_{d} & \text { Deterministic part of } \dot{\mathbf{X}} \\ \mathbf{X}_{d} & \text { Deterministic part of } \mathbf{X} \\ y & \text { Axial direction coordinate of the cross-section } \\ z & \text { Distance to neutral axis of the cross-section } \\ \sigma_{z}(y, \mathbf{B}, t) & \text { Normal stress of } z \text { line of } y \text { cross-section } \\ \sigma_{z \max }(y, \mathbf{B}, t) & \text { Maximum normal stress of } y \text { cross-section }\end{array}$

\section{Introduction}

The beam is the common structural element of the civil and mechanical engineering. It could withstand the bending moment and shear force primarily. If the beam failure occurs during service, it should lead to the huge economic lost or the casualties. The failure due to fatigue might be the common one. In mechanical engineering, there are a lot of factors which result in the beam with random parameters. The factors should include manufacturing error of machine tool, ambient temperature, random vibration of the foundation of machine tool, material uniformity and so on. Moreover, many beams used in mechanical engineering commonly carry the cyclic load, such as the loads due to all kinds of faults of the rotating machine. Therefore, it is important to estimate the life of the beam with random parameters and subjected to the cyclic load.

The random parameters of the beam result in the random stress under the deterministic cyclic load. The known methods of estimating the life of metallic structures and components under random stress can be classified into two groups: time-domain method based on a cycle counting method and frequency-domain method using power spectral density (PSD) of stochastic processes [1]. One of time-domain method, the combination of rainflow counting and Palmgren-Miner rule, is recognized as the "gold standard" for estimating fatigue damage from variable amplitude stress or for comparison to other estimate techniques $[2,3]$. However, it has been proved to spend a lot of time in processing data when time-domain method is employed to estimate the life under random stress $[4,5]$. For the reason, frequency-domain method has been developed. It can produce (exact or approximated) analytical expressions by which we can estimate cycle distribution and fatigue life under a given counting procedure, without, however, requiring knowledge of each stress or strain time history $[6,7]$. The life estimation of the beam under random stress has reported by some documents. The focus of the reported works is to predict the life of the beam with the deterministic parameters and withstanding the random loading. For example, the fatigue life prediction method of the dual cantilever beam subjected to the narrow band random load was introduced by Rajnikant Bhogilal Thakkar [8] and was based on the fatigue life under the variable sinusoidal load. The fatigue life of the Aluminum 6061 T6 cantilever beam, where the input was the white noise acceleration, were predicted using seven types of the frequency methods and Rainflow counting method respectively and they were compared with these obtained by experiments in [9]. The effect of the damping ratios of the test structure on the fatigue life was also analyzed by the four types of tests with the different damping ratios. An analytic methodology 
was developed by Paulus et al. [10] to predict the fatigue life of the cantilever beam experiencing stationary and Gaussian random vibration excitation. In addition, Paulus et al. [11] proposed a semi-empirical life method of the cantilevered beam subjected to the random excitation with a wide range of PSD (power spectral density) shapes and amplitudes. Two methods were employed by Lin et al. $[12,13]$ to predict the fatigue lives of RC beams strengthened with CFL (carbon fiber laminate) subjected to the exciting force due to the random traffic flow. They were based on the residual life model and the residual strength model respectively. Moreover, the fatigue life of the beam with the uncertain parameters was discussed by a few works. For example, the effect of the uncertainties on the fatigue life of a higher order sandwich beam was discussed by Wang and Sun [14]. In their work, the uncertainties included the density of foam core materials and the thicknesses of the core and the top bottom skins, two independent white noise loadings with the same spectral density function were applied to two different points on the top skin.

It has not been reported to estimate the fatigue life of the beam which has several random parameters and is subjected to cyclic load. Therefore, it should be discussed in this work. Here, these random parameters are assumed to be normally distributed. The remainder of this paper is organized as follows: In Section 2, it should be tested by Monte-Carlo simulation that the generalized displacement and velocity follow normal distributions with the periodic mean and variance estimated by the random perturbation method, and the mean, variance and correlation coefficient of the random dynamic stress and its time derivative are formulated using the first-order approximation in a Taylor series. In Section 3, the expected cumulative damage based on Palmgren-Miner rule is modeled where the random dynamic stress is non-stationary and follows the normal distribution with the nonzero mean at any time $t$. The conclusions are drawn finally.

\section{Random dynamical stress analysis of the beam with normal distribution parameters}

\subsection{Random dynamical response analysis}

When the beam undergoes small random vibrations around a position of stable equilibrium and is assumed to be with viscous damping, the dynamical equation of the beam with random parameters is:

$$
\mathbf{M} \ddot{\mathbf{X}}+\mathbf{C} \dot{\mathbf{X}}+\mathbf{K X}=\mathbf{F}(t),
$$

where $\mathbf{M}, \mathbf{C}$ and $\mathbf{K}$ are the $n \times n$ dimension mass matrix, damping matrix and stiffness matrix respectively and $\mathbf{F}(t)$ is the $n$ dimension external load vector of which the element is the cyclic external load. $\ddot{\mathbf{X}}, \dot{\mathbf{X}}$ and $\mathbf{X}$ are the $n$ dimension generalized acceleration, velocity and displacement vector respectively. $n$ is the total number of the degree of freedom. One or multiple elements of $\mathbf{M}, \mathbf{C}$ and $\mathbf{K}$ are the random variables. All random parameters of the considered beam are written by one random vector B. Generally, B is composed of the total length $L$, width $b$, height $h$, modulus of elasticity $E$ and density $\rho$ which are supposed to be normally distributed and mutually independent. The random perturbation method $[15,16]$ is employed to analyze the random dynamical response of the dynamical Eq. (1). The mean of the generalized displacement vector is:

$E[\mathbf{X}(t)]=E\left[\mathbf{X}_{d}(t)\right]=\mathbf{X}_{d}(t)$,

where $\mathbf{X}_{d}(t)$ is the deterministic part of the generalized displacement vector $\mathbf{X}$ and is solved by:

$$
\mathbf{M}_{d} \ddot{\mathbf{X}}_{d}+\mathbf{C}_{d} \dot{\mathbf{X}}_{d}+\mathbf{K}_{d} \mathbf{X}_{d}=\mathbf{F}(t),
$$

where $\mathbf{M}_{d}, \mathbf{C}_{d}$ and $\mathbf{K}_{d}$ are the deterministic part of $\mathbf{M}, \mathbf{C}$ and $\mathbf{K}$ respectively. They are obtained by substituting the mean of all random parameters into $\mathbf{M}, \mathbf{C}$ and $\mathbf{K}$. The covariance matrix of the 
generalized displacement vector is:

$\operatorname{Cov}(\mathbf{X})=\left[\begin{array}{cccc}\operatorname{Var}(x(1)) & & & \text { symmetry } \\ \operatorname{Cov}(x(2), x(1)) & \operatorname{Var}(x(2)) & & \\ \vdots & \vdots & \ddots & \\ \operatorname{Cov}(x(n), x(1)) & \operatorname{Cov}(x(n), x(2)) & \cdots & \operatorname{Var}(x(n))\end{array}\right]=\mathbf{X}_{\mathbf{B}} \operatorname{Cov}(\mathbf{B}) \mathbf{X}_{\mathbf{B}}^{\mathrm{T}}$,

where $\mathbf{X}_{\mathbf{B}}$ is the sensitivity matrix of $\mathbf{X}_{d}(t)$ to the random vector $\mathbf{B}$ and is:

$\mathbf{X}_{\mathbf{B}}=\left[\begin{array}{cccc}x_{d, 1}(1) & x_{d, 2}(1) & \cdots & x_{d, m}(1) \\ x_{d, 1}(2) & x_{d, 2}(2) & \cdots & x_{d, m}(2) \\ \vdots & \vdots & & \vdots \\ x_{d, 1}(n) & x_{d, 2}(n) & \cdots & x_{d, m}(n)\end{array}\right]$

$\operatorname{Cov}(\mathbf{B})$ is the covariance matrix of the random parameter vector $\mathbf{B}$ and is:

$\operatorname{Cov}(\mathbf{B})=\left[\begin{array}{cccc}\operatorname{Var}\left(b_{1}\right) & & & \text { symmetry } \\ \operatorname{Cov}\left(b_{2}, b_{1}\right) & \operatorname{Var}\left(b_{2}\right) & & \\ \vdots & \vdots & \ddots & \\ \operatorname{Cov}\left(b_{m}, b_{1}\right) & \operatorname{Cov}\left(b_{m}, b_{2}\right) & \cdots & \operatorname{Var}\left(b_{m}\right)\end{array}\right]$,

where $m$ is the total number of random parameters and $x_{d, j}(i)=\partial x_{d}(i) / \partial b_{j}$ which is solved by:

$$
\begin{aligned}
& \mathbf{M}_{d} \ddot{\mathbf{X}}_{d, j}+\mathbf{C}_{d} \dot{\mathbf{X}}_{d, j}+\mathbf{K}_{d} \mathbf{X}_{d, j}=-\left(\mathbf{M}_{d, j} \ddot{\mathbf{X}}_{d}+\mathbf{C}_{d, j} \dot{\mathbf{X}}_{d}+\mathbf{K}_{d, j} \mathbf{X}_{d}\right) . \\
& \mathbf{M}_{d, j}=\left.\frac{\partial \mathbf{M}}{\partial b_{j}}\right|_{\mathbf{B}=\mathbf{B}_{d}}, \quad \mathbf{C}_{d, j}=\left.\frac{\partial \mathbf{C}}{\partial b_{j}}\right|_{\mathbf{B}=\mathbf{B}_{d}}, \quad \mathbf{K}_{d, j}=\left.\frac{\partial \mathbf{K}}{\partial b_{j}}\right|_{\mathbf{B}=\mathbf{B}_{d}},
\end{aligned}
$$

where $\mathbf{B}_{d}$ is the deterministic part of $\mathbf{B}$.

The skewness and kurtosis of the generalized displacement and velocity of node 1, 3 and 5 of the random cantilever beam at 0.005 and 0.012 second are shown in table 1 . All random parameters' $C V$ is the identical number in one Monte-Carlo simulation. The samples of the generalized displacement are generated by Monte-Carlo method and Newmark- $\beta$ method. The sample size is 5000 . The considered cantilever beam with normal distribution parameters is shown in Fig. 1(b). It is divided into 5 elements. The means of the total length $L$, width $b$, height $h$, density $\rho_{1}$ and elastic modulus $E$ are $0.25 \mathrm{~m}, 0.02 \mathrm{~m}, 0.03 \mathrm{~m}, 7850 \mathrm{~kg} / \mathrm{m}^{3}$ and $2.06 \times 10^{11} \mathrm{~Pa}$ respectively. The deterministic cyclic load $F(t)=3500 \sin (100 \pi t)$ is applied to the free end node. From Table 1, it could be trusted that the random generalized displacement and velocity at any time are the normal distribution when all $\mathrm{CVs}$ of the random parameters are small (generally $C V \leq 0.01)$.

The displacement mean and standard deviation of node 1 of the cantilever beam with normal distribution parameters calculated by Monte-Carlo simulation and random perturbation method respectively with $C V=0.01$ are shown in Fig. 2 and 3. Here, the values of the parameters except $\mathrm{CV}$ are identical with the numerical calculation of the skewness and kurtosis of the generalized displacement and velocity. The difference between Monte-Carlo simulation and random perturbation method is very little according to Fig. 2 and 3. In fact, the similar results are found in the mean and standard deviation of the generalized displacement and velocity of other nodes. Therefore, it might be one solution to estimating the mean and variance by random perturbation method when the random generalized displacement and velocity of the beam with normal distribution parameters are normally distributed at any time. 
Table 1. Skewness and kurtosis of generalized displacement and velocity of node 1, 3 and 5 of cantilever beam with normal distribution random parameters at 0.005 and 0.012 second

\begin{tabular}{|c|c|c|c|c|c|c|c|c|c|c|c|c|c|c|}
\hline \multirow{2}{*}{$C V$} & \multirow{2}{*}{$\begin{array}{l}\text { Time } \\
\text { (s) }\end{array}$} & & \multicolumn{4}{|c|}{ Node 1} & \multicolumn{4}{|c|}{ Node 3} & \multicolumn{4}{|c|}{ Node 5} \\
\hline & & & $x(1)$ & $\dot{\dot{x}}(1)$ & $x(2)$ & $\dot{x}(2)$ & $x(5)$ & $\dot{x}(5)$ & $x(6)$ & $\dot{x}(6)$ & $x(9)$ & $\dot{x}(9)$ & $x(10)$ & $\dot{x}(10)$ \\
\hline \multirow{4}{*}{.00} & \multirow{2}{*}{0.005} & & 0.060 & 0.072 & .057 & 0.067 & 0.060 & 0.071 & 0.057 & 0.066 & .060 & 0.071 & .057 & \\
\hline & & $\mathrm{Ku}$ & 3.007 & 3.010 & 3.004 & 3.007 & 3.007 & 3.009 & 3.004 & 3.007 & 3.007 & 3.009 & 004 & 07 \\
\hline & \multirow{2}{*}{0.012} & & 0.063 & -0.053 & 0.059 & 0.052 & 0.062 & 54 & 0.059 & -0 . & 62 & 54 & 59 & -0.053 \\
\hline & & & 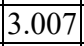 & & t & & & & & & .007 & & 3.005 & \\
\hline \multirow{4}{*}{0} & \multirow[b]{2}{*}{0} & & & & & & & & & & & & & \\
\hline & & $\mathrm{Ku}$ & 019 & 27 & .073 & \begin{tabular}{|l|}
3.070 \\
\end{tabular} & 019 & 3.027 & 3.073 & 3.070 & 3.019 & 3.026 & .073 & 3.070 \\
\hline & \multirow{2}{*}{0.012} & Ske & 0.153 & -0.135 & 0.150 & -0.131 & 0.153 & -0.136 & 0.150 & -0.132 & 0.153 & -0.137 & 0.149 & -0.134 \\
\hline & & & 3.021 & 3.017 & 3.072 & 3.084 & 3.020 & 3.017 & 3.073 & 3.082 & 3.020 & 3.017 & 3.073 & 3.081 \\
\hline \multirow{4}{*}{0.0} & \multirow{2}{*}{0.005} & & 744 & 0.882 & 0.712 & 0.834 & .744 & 0.876 & 0.713 & 0.825 & 0.744 & 0.870 & 0.713 & 0.817 \\
\hline & & & 4.138 & 4.541 & 4.056 & 4.413 & 134 & 4.522 & 4.057 & 4.383 & 4.135 & 4.563 & 4.058 & 4.362 \\
\hline & \multirow{2}{*}{0.012} & Ske & 0.776 & -0.681 & 0.740 & -0.662 & 0.775 & -0.686 & 0.738 & -0.668 & 0.773 & -0.690 & 0.737 & -0.673 \\
\hline & & Kur & 4.229 & 3.999 & 4.140 & 3.936 & 4.225 & 4.009 & 4.133 & 3.952 & 4.220 & 4.019 & 4.128 & 3.963 \\
\hline
\end{tabular}

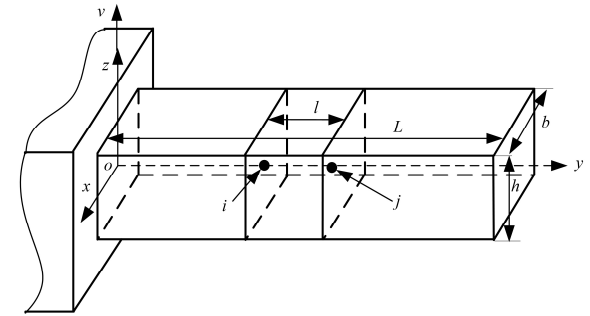

a)

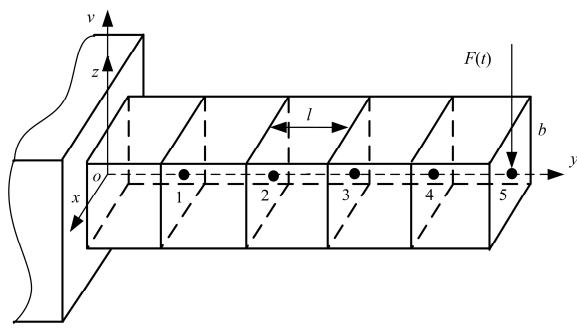

b)

Fig. 1. a) Coordinate system and b) finite element model of the beam

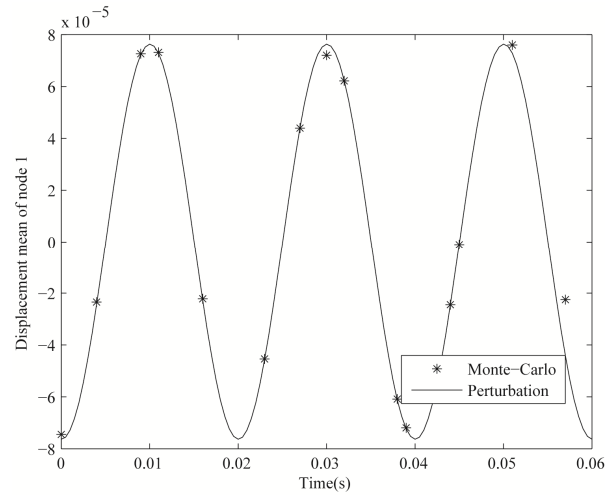

Fig. 2. Displacement mean of node 1 of the considered cantilever beam with $C V=0.01$

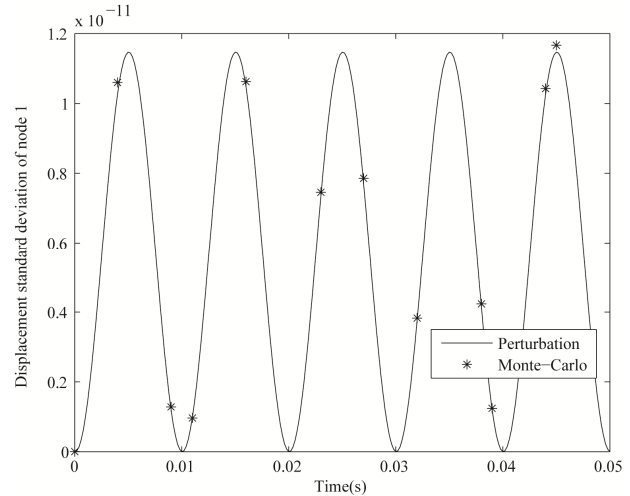

Fig. 3. Displacement standard deviation of node 1 of the considered cantilever beam with $C V=0.01$

\subsection{Random dynamical stress analysis}

The coordinate system of the considered beam is shown in Fig. 1(a). The normal stress of a cross-section of the Euler-Bernoulli beam for the application of bending moment and transverse force is [17]:

$\sigma_{z}(y, \mathbf{B}, t)=-\frac{M(y, \mathbf{B}, t) z}{I(y, \mathbf{B})}$

where $y$ is the axial direction coordinate of the cross-section, $M(y, \mathbf{B}, t)$ is the bending moment 
of the cross-section at the time $t, I(y, \mathbf{B})$ is area moment of inertia and $z$ is the distance to neutral axis of the cross-section. By substituting the approximate differential equation of the deflection curve of the beam into Eq. (8), the normal stress is:

$\sigma_{z}(y, \mathbf{B}, t)=-E z \frac{\mathrm{d}^{2} v(y, \mathbf{B}, t)}{\mathrm{d} y^{2}}$

where $E, v(y, \mathbf{B}, t)$ are the modulus of elasticity and the deflection of the cross-section $y$ at the time $t$ respectively. The maximal normal stress of the cross-section $y$ at the time $t$ is:

$\sigma_{z \max }(y, \mathbf{B}, t)=E \frac{h}{2} \frac{\mathrm{d}^{2} v(y, \mathbf{B}, t)}{\mathrm{d} y^{2}}$.

In the finite element method for the Euler-Bernoulli beam, $\mathrm{d}^{2} v(y, \mathbf{B}, t) / \mathrm{d} y^{2}$ between any two adjacent node $i$ and $j(i, j=1,2,3, \ldots, i<j$ and $j \leq n / 2)$ is:

$$
\begin{aligned}
& \frac{d^{2} v(y, \mathbf{B}, t)}{d y^{2}}=2\left[\frac{3}{l^{2}}\left(x_{2 j-1}-x_{2 i-1}\right)-\frac{1}{l}\left(2 x_{2 i}+x_{2 j}\right)\right] \\
& \quad+6 y\left[\frac{2}{l^{3}}\left(x_{2 i-1}-x_{2 j-1}\right)+\frac{1}{l^{2}}\left(x_{2 i}+x_{2 j}\right)\right]
\end{aligned}
$$

where the $y$-coordinate of the node $j$ is more than that of the node $i, x_{2 i-1}, x_{2 i}, x_{2 j-1}$ and $x_{2 j}$ are the lateral displacement and rotation of the node $i$ and $j$ respectively and $l$ is the element length. According to Eq. (11), the maximal normal stress of the cross-section $y$ at the time $t$ is normally distributed if $x_{2 i-1}, x_{2 j-1}, x_{2 i}$ and $x_{2 i}$ are normally distributed. Then, by expanding the maximal normal stress of the cross-section $y$ to first-order approximation in a Taylor series at a point $\mathbf{B}=\mathbf{B}_{d}$, the mean and variance are respectively calculated by [18]:

$E\left[\sigma_{z \max }(y, \mathbf{B}, t)\right]=\sigma_{z \max }\left(y, \mathbf{B}_{d}, t\right)$,

$\operatorname{Var}\left[\sigma_{z \max }(y, \mathbf{B}, t)\right]=\left[\left.\frac{\partial \sigma_{z \max }(y, \mathbf{B}, t)}{\partial \mathbf{B}}\right|_{\mathbf{B}=\mathbf{B}_{d}}\right]^{[2]} \operatorname{Var}(\mathbf{B})$,

where:

$\operatorname{Var}(\mathbf{B})$

$=\left[\operatorname{Var}\left(b_{1}\right), \operatorname{Cov}\left(b_{1}, b_{2}\right), \operatorname{Cov}\left(b_{1}, b_{3}\right), \ldots, \operatorname{Cov}\left(b_{1}, b_{m}\right), \operatorname{Cov}\left(b_{2}, b_{1}\right), \operatorname{Var}\left(b_{2}\right), \operatorname{Cov}\left(b_{2}, b_{3}\right), \ldots, \operatorname{Var}\left(b_{m}\right)\right]^{\mathrm{T}}$,

is $m^{2} \times 1$ vector and:

$$
\begin{gathered}
{\left[\left.\frac{\partial \sigma_{z \max }(y, \mathbf{B}, t)}{\partial \mathbf{B}}\right|_{\mathbf{B}=\mathbf{B}_{d}}\right]^{[2]}} \\
=\left[\left(\left.\frac{\partial \sigma_{z \max }(y, \mathbf{B}, t)}{\partial b_{1}}\right|_{\mathbf{B}=\mathbf{B}_{d}}\right)^{2},\left.\frac{\partial \sigma_{z \max }(y, \mathbf{B}, t)}{\partial b_{1}} \frac{\partial \sigma_{z \max }(y, \mathbf{B}, t)}{\partial b_{2}}\right|_{\mathbf{B}=\mathbf{B}_{d}},\right. \\
\quad \ldots,\left.\quad \frac{\partial \sigma_{z \max }(y, \mathbf{B}, t)}{\partial b_{1}} \frac{\partial \sigma_{z \max }(y, \mathbf{B}, t)}{\partial b_{m}}\right|_{\mathbf{B}=\mathbf{B}_{d}},\left.\frac{\partial \sigma_{z \max }(y, \mathbf{B}, t)}{\partial b_{2}} \frac{\partial \sigma_{z \max }(y, \mathbf{B}, t)}{\partial b_{1}}\right|_{\mathbf{B}=\mathbf{B}_{d}}, \\
\left.\left(\left.\frac{\partial \sigma_{z \max }(y, \mathbf{B}, t)}{\partial b_{2}}\right|_{\mathbf{B}=\mathbf{B}_{d}}\right)^{2}, \ldots,\left(\left.\frac{\partial \sigma_{z \max }(y, \mathbf{B}, t)}{\partial b_{m}}\right|_{\mathbf{B}=\mathbf{B}_{d}}\right)^{2}\right] .
\end{gathered}
$$


According to the random perturbation method and Eq. (10) and (11), the partial derivative of the maximal normal stress of the cross-section $y$ with respect to the random variable $b_{s}$ $(s=1,2, \ldots, m)$ between any two adjacent node $i$ and $j$ :

$$
\begin{aligned}
& \frac{\partial \sigma_{z \max }(y, \mathbf{B}, t)}{\partial b_{s}}=\frac{\partial(E h / 2)}{\partial b_{s}} \frac{\mathrm{d}^{2} x}{\mathrm{~d} y^{2}}+E \frac{h}{2}\left\{2 \left[\frac{\partial\left(3 / l^{2}\right)}{\partial b_{s}}\left(x_{2 j-1}-x_{2 i-1}\right)\right.\right. \\
& \left.+\frac{3}{l^{2}}\left(\frac{\partial x_{2 j-1}}{\partial b_{s}}-\frac{\partial x_{2 i-1}}{\partial b_{s}}\right)-\frac{\partial(1 / l)}{\partial b_{s}}\left(2 x_{2 i}+x_{2 j}\right)-\frac{1}{l}\left(2 \frac{\partial x_{2 i}}{\partial b_{s}}+\frac{\partial x_{2 j}}{\partial b_{s}}\right)\right] \\
& +6 y\left[\frac{\partial\left(2 / l^{3}\right)}{\partial b_{s}}\left(x_{2 i-1}-x_{2 j-1}\right)+\frac{2}{l^{3}}\left(\frac{\partial x_{2 i-1}}{\partial b_{s}}-\frac{\partial x_{2 j-1}}{\partial b_{s}}\right)\right. \\
& \left.\left.+\frac{\partial\left(1 / l^{2}\right)}{\partial b_{s}}\left(x_{2 i}+x_{2 j}\right)+\frac{1}{l^{2}}\left(\frac{\partial x_{2 i}}{\partial b_{s}}+\frac{\partial x_{2 j}}{\partial b_{s}}\right)\right]\right\}
\end{aligned}
$$

where $\partial x_{2 j-1} / \partial b_{s}=x_{d, s}(2 j-1), \quad \partial x_{2 i-1} / \partial b_{s}=x_{d, s}(2 i-1), \quad \partial x_{2 j} / \partial b_{s}=x_{d, s}(2 j)$ and $\partial x_{2 i} / \partial b_{s}=x_{d, s}(2 i)$.

By combining Eqs. (10) and (11), the time derivative of the maximal normal stress of the cross-section $y$ is:

$$
\begin{aligned}
& \dot{\sigma}_{z \max }(y, \mathbf{B}, t)=E \frac{h}{2}\left\{2\left[\frac{3}{l^{2}}\left(\dot{x}_{2 j-1}-\dot{x}_{2 i-1}\right)-\frac{1}{l}\left(2 \dot{x}_{2 i}+\dot{x}_{2 j}\right)\right]\right. \\
& \left.+6 y\left[\frac{2}{l^{3}}\left(\dot{x}_{2 i-1}-\dot{x}_{2 j-1}\right)+\frac{1}{l^{2}}\left(\dot{x}_{2 i}+\dot{x}_{2 j}\right)\right]\right\} .
\end{aligned}
$$

Then, its approximate mean and variance could be calculated by Eqs. (12-14) where $\sigma_{z \max }(y, \mathbf{B}, t)$ is replaced by $\dot{\sigma}_{z \max }(y, \mathbf{B}, t)$ and $\partial \dot{\sigma}_{z \max }(y, \mathbf{B}, t) / \partial b_{s}=\mathrm{d}\left[\partial \sigma_{z \max }(y, \mathbf{B}, t) / \partial b_{s}\right] / \mathrm{d} t$. According to Eq. (16), the time derivative of the maximal normal stress of the cross-section $y$ at the time $t$ is normally distributed if $\dot{x}_{2 i-1}, \dot{x}_{2 i}, \dot{x}_{2 j-1}$ and $\dot{x}_{2 j}$ are normally distributed. By expanding the maximal normal stress of the cross-section $y$ and its time derivative to first-order approximation in a Taylor series at a point $\mathbf{B}=\mathbf{B}_{d}, \sigma_{z \max }(y, \mathbf{B}, t)-\sigma_{z \max }\left(y, \mathbf{B}_{d}, t\right)$ and $\dot{\sigma}_{z \max }(y, \mathbf{B}, t)-\dot{\sigma}_{z \max }\left(y, \mathbf{B}_{d}, t\right)$ respectively are:

$$
\begin{aligned}
& \sigma_{z \max }(y, \mathbf{B}, t)-\sigma_{z \max }\left(y, \mathbf{B}_{d}, t\right)=\left.\sum_{i=1}^{m} \frac{\partial \sigma_{z \max }(y, \mathbf{B}, t)}{\partial b_{i}}\right|_{\mathbf{B}=\mathbf{B}_{d}}\left(b_{i}-b_{i, d}\right), \\
& \dot{\sigma}_{z \max }(y, \mathbf{B}, t)-\dot{\sigma}_{z \max }\left(y, \mathbf{B}_{d}, t\right)=\left.\sum_{j=1}^{m} \frac{\partial \dot{\sigma}_{z \max }(y, \mathbf{B}, t)}{\partial b_{j}}\right|_{\mathbf{B}=\mathbf{B}_{d}}\left(b_{j}-b_{j, d}\right),
\end{aligned}
$$

where $b_{i, d}$ and $b_{j, d}$ are the mean of the random variables $b_{i}$ and $b_{j}$ respectively. Then, the correlation coefficient between $\sigma_{z \max }(y, \mathbf{B}, t)$ and $\dot{\sigma}_{z \max }(y, \mathbf{B}, t)$ is:

$$
\begin{aligned}
\rho(y, t) & \\
= & \frac{E\left[\left(\left.\sum_{i=1}^{m} \frac{\partial \sigma_{z \max }(y, \mathbf{B}, t)}{\partial b_{i}}\right|_{\mathbf{B}=\mathbf{B}_{d}}\left(b_{i}-b_{i, d}\right)\right)\left(\left.\sum_{j=1}^{m} \frac{\partial \dot{\sigma}_{z \max }(y, \mathbf{B}, t)}{\partial b_{j}}\right|_{\mathbf{B}=\mathbf{B}_{d}}\left(b_{j}-b_{j, d}\right)\right)\right]}{\sqrt{\operatorname{Var}\left[\dot{\sigma}_{z \max }(y, \mathbf{B}, t)\right] \operatorname{Var}\left[\sigma_{z \max }(y, \mathbf{B}, t)\right]}} \\
= & \frac{\left.\sum_{i=1}^{m} \sum_{j=1}^{m} \frac{\partial \sigma_{z \max }(y, \mathbf{B}, t)}{\partial b_{i}} \frac{\partial \dot{\sigma}_{z \max }(y, \mathbf{B}, t)}{\partial b_{j}}\right|_{\mathbf{B}=\mathbf{B}_{d}} \operatorname{Cov}\left(b_{i}, b_{j}\right)}{\sqrt{\operatorname{Var}\left[\dot{\sigma}_{z \max }(y, \mathbf{B}, t)\right] \operatorname{Var}\left[\sigma_{z \max }(y, \mathbf{B}, t)\right]}},
\end{aligned}
$$


The mean and standard deviation of the maximal fixed-end stress of the cantilever beam calculated using the presented method are compared with these by Monte-Carlo simulation in Fig. 4 and 5 respectively. The correlation coefficient between the maximal fixed-end stress and its time derivative calculated by Monte-Carlo simulation and Eq. (19) is shown in Fig. 6. Here, $C V=0.005$ and other parameters are the same as Section 2.1. It could be seen that the mean, standard deviation and correlation coefficient estimated by the presented method are very close to these calculated by Monte-Carlo simulation from Figs. 4, 5 and 6. In fact, there is only a small difference between the mean, standard deviation and correlation coefficient estimated by the presented method and Monte-Carlo simulation when $C V$ of all random parameters is less than about 0.01 .

\section{Life estimation of a beam with normal distribution parameters and subjected to cyclic load}

According to Palmgren-Miner rule, the expected cumulative damage during the time interval $t_{1}$ to $t_{2}$ is [19]:

$E\left[D\left(t_{1}, t_{2}\right)\right]=-\frac{1}{\mathrm{C}} \int_{t_{1}}^{t_{2}} \int_{0}^{\infty} \frac{\partial \bar{v}_{\sigma_{z \max }}^{+}(t)}{\partial \sigma_{z \max }} \sigma_{z \max }^{m} d \sigma_{z \max } d t$,

where $C$ and $m$ are constants depending on the material, $\sigma_{z \max }(y, \mathbf{B}, t)$ and $\dot{\sigma}_{z \max }(y, \mathbf{B}, t)$ are replaced by $\sigma_{z \max }$ and $\dot{\sigma}_{z \max }$ respectively for simplicity, $\bar{v}_{\sigma_{z \max }}(t)$ is the expected number of crossings (at level $\sigma_{z \max }$, with positive slope) and is calculated by [20]:

$\bar{v}_{\sigma_{z \max }(y, \mathbf{B}, t)}^{+}(t)=\int_{0}^{\infty} \dot{\sigma}_{z \max } p\left(\sigma_{z \max }, t ; \dot{\sigma}_{z \max }, t\right) d \dot{\sigma}_{z \max }$.

If $\sigma_{z \max }$ and $\dot{\sigma}_{z \max }$ are normally distributed, their joint probability density function is:

$$
\begin{aligned}
& p\left(\sigma_{z \max }, t ; \dot{\sigma}_{z \max }, t\right) \\
& \exp \left[-\frac{1}{2 \sqrt{1-\rho^{2}}}\left(\begin{array}{c}
\left.\frac{\left(\sigma_{z \max }-\bar{\sigma}_{z \max }\right)^{2}}{\operatorname{Var}\left(\sigma_{z \max }\right)}-\frac{2 \rho\left(\sigma_{z \max }-\bar{\sigma}_{z \max }\right)\left(\dot{\sigma}_{z \max }-\dot{\dot{\sigma}}_{z \max }\right)}{\sqrt{\operatorname{Var}\left(\sigma_{z \max }\right) \operatorname{Var}\left(\dot{\sigma}_{z \max }\right)}}\right) \\
+\frac{\left(\dot{\sigma}_{z \max }-\overline{\dot{\sigma}}_{z \max }\right)^{2}}{\operatorname{Var}\left(\dot{\sigma}_{z \max }\right)}
\end{array}\right)\right]
\end{aligned}
$$

where $\rho=\rho(y, t)$ is estimated by Eq. (19), $\bar{\sigma}_{z \max }=\sigma_{z \max }\left(y, \mathbf{B}_{d}, t\right)$ and $\overline{\dot{\sigma}}_{z \max }=\dot{\sigma}_{z \max }\left(y, \mathbf{B}_{d}, t\right)$. Then, the expected number of crossings is:

$$
\begin{aligned}
& \bar{v}_{\sigma_{z \max }}^{+}(t)=\frac{\exp \left(-\frac{\left(\sigma_{z \max }-\bar{\sigma}_{z \max }\right)^{2}}{2 \operatorname{Var}\left(\sigma_{z \max }\right)}\right)}{2 \pi \operatorname{Var}\left(\sigma_{z \max }\right) \sqrt{2\left(1-\rho^{2}\right)}}\left\{\frac{\sqrt{2 \operatorname{Var}\left(\sigma_{z \max }\right) \operatorname{Var}\left(\dot{\sigma}_{z \max }\right)}\left(1-\rho^{2}\right)}{\exp \left(\varphi^{2}\right)}\right. \\
& \left.+\sqrt{\pi\left(1-\rho^{2}\right)}\left[\bar{\sigma}_{z \max } \sqrt{\operatorname{Var}\left(\sigma_{z \max }\right)}+\rho \sqrt{\operatorname{Var}\left(\dot{\sigma}_{z \max }\right)}\left(\sigma_{z \max }-\bar{\sigma}_{z \max }\right)\right] \operatorname{Erfc}(-\varphi)\right\},
\end{aligned}
$$

where $\operatorname{Erfc}(z)=1-\operatorname{erf}(z), \operatorname{erf}(z)$ is the error function defined by:

$\operatorname{erf}(z)=\frac{2}{\sqrt{\pi}} \int_{0}^{z} \exp \left(-t^{2}\right) d t$ 
and:

$\varphi=\frac{\overline{\dot{\sigma}}_{z \max } \sqrt{\operatorname{Var}\left(\sigma_{z \max }\right)}+\rho \sqrt{\operatorname{Var}\left(\dot{\sigma}_{z \max }\right)}\left(\sigma_{z \max }-\bar{\sigma}_{z \max }\right)}{\sqrt{2\left(1-\rho^{2}\right) \operatorname{Var}\left(\sigma_{z \max }\right) \operatorname{Var}\left(\dot{\sigma}_{z \max }\right)}}$.

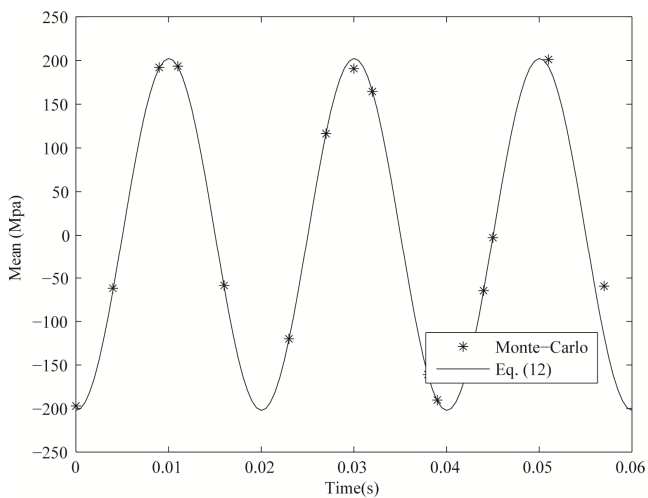

Fig. 4. Mean of the maximal fixed-end stress of the considered cantilever beam when $C V=0.005$ and other parameters are the same as Section 2.1

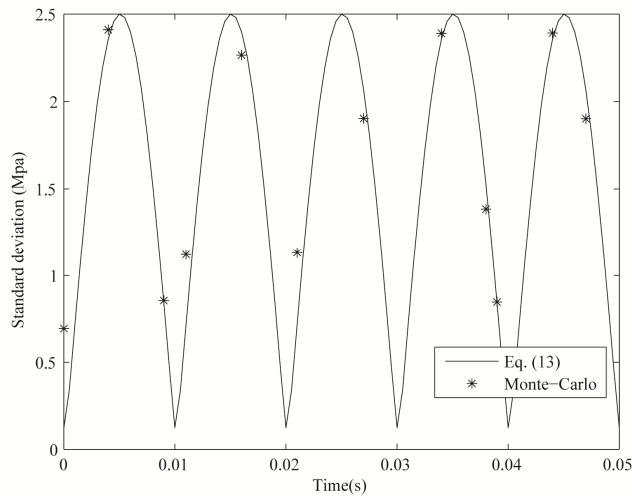

Fig. 5. Standard deviation of the maximal fixed-end stress of the considered cantilever beam when $C V=0.005$ and other parameters are the same as Section 2.1

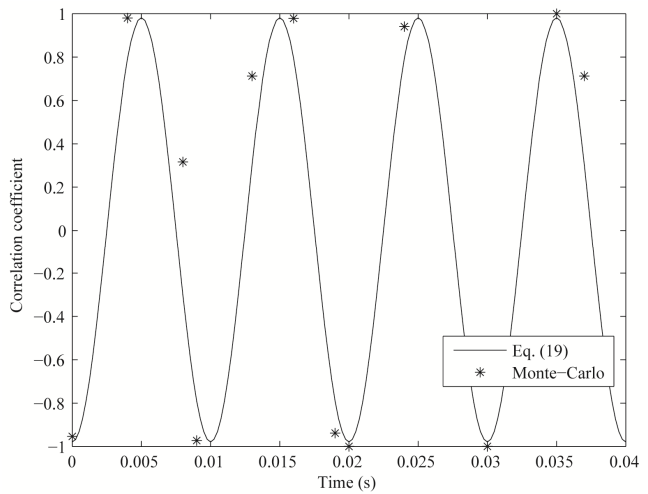

Fig. 6. Correlation coefficient between maximal fixed-end stress and its time derivative of the considered cantilever beam when $C V=0.005$ and other parameters are the same as Section 2.1

Then, the partial derivative of the expected number of crossings with respect to $\sigma_{z \max }$ is:

$$
\begin{aligned}
& \frac{\partial \bar{v}_{\sigma_{\text {max }}}^{+}(t)}{\partial \sigma_{z \max }}=\frac{\exp \left(-\frac{\left(\sigma_{z \max }-\bar{\sigma}_{z \max }\right)^{2}}{2 \operatorname{Var}\left(\sigma_{z \max }\right)}\right)}{2 \sqrt{2} \pi\left[\operatorname{Var}\left(\sigma_{z \max }\right)\right]^{2}} \\
& \quad \times\left\{\sqrt{2\left(1-\rho^{2}\right) \operatorname{Var}\left(\sigma_{z \max }\right) \operatorname{Var}\left(\dot{\sigma}_{z \max }\right)}\left(\bar{\sigma}_{z \max }-\sigma_{z \max }\right) \exp \left(-\varphi^{2}\right)-\lambda[1+\operatorname{Erfc}(-\varphi)]\right\},
\end{aligned}
$$

where:

$$
\begin{aligned}
\lambda= & \sqrt{\pi}\left[\rho \sqrt{\operatorname{Var}\left(\dot{\sigma}_{z \max }\right)} \bar{\sigma}_{z \max }^{2}+\overline{\dot{\sigma}}_{z \max } \sigma_{z \max } \sqrt{\operatorname{Var}\left(\sigma_{z \max }\right)}+\rho \sqrt{\operatorname{Var}\left(\dot{\sigma}_{z \max }\right)}\right. \\
& \left.\times\left(\sigma_{z \max }^{2}-\bar{\sigma}_{z \max }^{2}\right)-\bar{\sigma}_{z \max }\left(\overline{\dot{\sigma}}_{z \max } \sqrt{\operatorname{Var}\left(\sigma_{z \max }\right)}+2 \rho \sigma_{z \max } \sqrt{\operatorname{Var}\left(\dot{\sigma}_{z \max }\right)}\right)\right] .
\end{aligned}
$$

Then, the expected cumulative damage of the beam with normal distribution parameters and 
subjected to cyclic load during the time interval $t_{1}$ to $t_{2}$ could be calculated by substituting Eq. (25) and (26) into Eq. (20) and using the numerical integration method.

Using the proposed method, the expected instantaneous damages $\left(\int_{0}^{\infty} \partial \bar{v}_{\sigma_{z \max }}^{+}(t) / \partial \sigma_{z \max } \sigma_{z \max }^{m} d \sigma_{z \max }\right)$ of the cantilever beam with normal distribution parameters are shown in Fig. 7 and 8 when $C V=0.005$ and 0.010 respectively. Other parameters of the considered beam are the same as Section 2.1. The expected instantaneous damage of the discussed random cantilever beam subjected to cyclic load is the periodic function of the time $t$ and its amplitude with $C V=0.010$ is more than one with $C V=0.005$ from Fig. 7 and 8 . The expected cumulative damage with $C V=0.005$ and 0.010 during one second are $4.945 \times 10^{-4}$ and $1.742 \times 10^{-3}$ and their corresponding lives (numbers of cycles to failure) are $1.072 \times 10^{5}$ and $3.043 \times 10^{4}$ respectively.

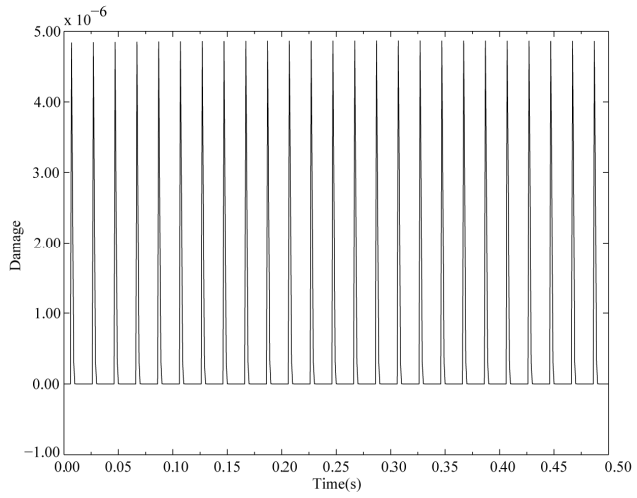

Fig. 7. Expected instantaneous damage of the cantilever beam with normal distribution parameters and subjected to cyclic load when $C V=0.005$ and other parameters are the same as Section 2.1

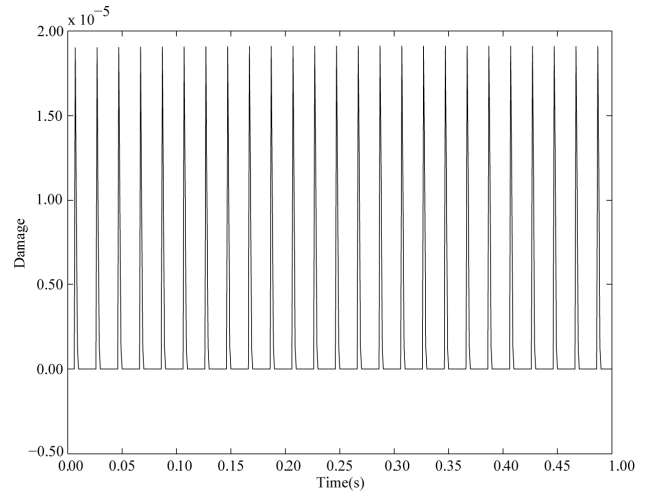

Fig. 8. Expected instantaneous damage of the cantilever beam with normal distribution parameters and subjected to cyclic load when $C V=0.01$ and other parameters are the same as Section 2.1

Li Changyou offers the idea and the most of the theoretical innovation of this article. The random dynamical response was analyzed and the most of the revision works of the manuscript were done by Wang Xuchu. The random dynamical stress was analyzed by Wang Wei. The prominent assistance in the theory analysis was offered by Zhang Yimin. The simulation of Section 3 was done by Guo Song.

\section{Conclusions}

One method for estimating the life of the beam with normal distribution parameters and subjected to cyclic load was presented in this work. The random generalized displacement and velocity of the beam with random parameters at any time are the normal distributions when CVs of all random parameters are small (generally $C V \leq 0.01$ ). Their means and variances could be accurately estimated by the random perturbation method. By compared with Monte-Carlo simulation, the means, variances and their correlation coefficient of the random dynamic stress and its time derivative could be estimated using the first-order approximation method in a Taylor series. The presented method could estimate the life of the beam with normal distribution parameters and subjected to cyclic load where the random dynamic stress is non-stationary and follows the normal distribution with the nonzero mean at any time $t$.

\section{Acknowledgements}

The work is supported by National Natural Science Foundation of China (Grant No. 51575095), Fundamental Research Funds for the Central Universities of China (Grant 
No. N140304006), China Ministry of Education New Century Excellent Person Support Plan (Grant No. NCET-12-0105) and Major State Basic Research Development Program of China (973 Program) (Grant No. 2014CB046303).

\section{References}

[1] Ge J., Sun Y., Zhou S. Fatigue life estimation under multiaxial random loading by means of the equivalent Lemaitre stress and multiaxial S-N curve methods. International Journal of Fatigue, Vol. 79, 2015, p. 65-74.

[2] Larsena C., Irvineb T. A review of spectral methods for variable amplitude fatigue prediction and new results. Procedia Engineering, Vol. 101, 2015, p. 243-250.

[3] Mrsnik M., Slavic J., Boltezar M. Frequency-domain methods for a vibration-fatigue-life estimation - application to real data. International Journal of Fatigue, Vol. 47, 2013, p. 8-17.

[4] Braccesi C., Cianetti F., Lori G., Pioli D. Random multiaxial fatigue: a comparative analysis among selected frequency and time domain fatigue evaluation methods. International Journal of Fatigue, Vol. 74, 2015, p. 107-118.

[5] Vidic G., Nagode M. Critical evaluation of frequency-domain approach for fatigue damage estimation. Journal of Engineering Materials and Technology, Vol. 136, Issue 3, 2014, p. 1-7.

[6] Benasciutti D., Tovo R. Spectral methods for lifetime prediction under wide-band stationary random processes. International Journal of Fatigue, Vol. 27, 2005, p. 867-877.

[7] He G., Chen H., He X. Fatigue behavior and influence factor analysis of the structure subject to multiaxial random loading. Journal of Vibroengineering, Vol. 17, Issue 7, 2015, p. 3620-3634.

[8] Thakkar R. Exact Sinusoidal Simulation of Fatigue under Gaussian Narrow Band Random Loading. Retrospective Theses and Dissertations, Iowa State University, 1972

[9] Eldogan Y., Cigeroglu E. Vibration fatigue analysis of a cantilever beam using different fatigue theories. Topics in Modal Analysis, Proceedings of the 31st IMAC, A Conference on Structural Dynamics, Conference Proceedings of the Society for Experimental Mechanics Series 45, Springer Verlag, Berlin, Vol. 7, 2013, p. 471-478.

[10] Paulus M., Dasgupta A., Habtour E. Life estimation model of a cantilevered beam subjected to complex random vibration. Fatigue and Fracture of Engineering Materials and Structures, Vol. 35, Issue 11, 2012, p. 1058-1070.

[11] Paulus M., Dasgupta A. Semi-empirical life model of a cantilevered beam subject to random vibration. International Journal of Fatigue, Vol. 45, 2012, p. 82-90.

[12] Lin R., Huang P., Zhao C., Guo X., Zheng X. Prediction of fatigue lives of RC beams strengthened with CFL under random loading. Acta Mechanica Solida Sinica, Vol. 21, Issue 4, 2008, p. 359-363.

[13] Lin R. The Methods for Predicting Fatigue Lives of RC Beams Strengthened with CFL Under Random Loads. Dissertation for Master's degree, South China University of Technology, Guangzhou, 2005.

[14] Wang X., Sun J. Random fatigue of a higher order sandwich beam with parameter uncertainties. Journal of Sound and Vibration, Vol. 260, Issue 2, 2003, p. 349-356.

[15] Chen P., Soroka W. Impulse response of a dynamic system with statistical properties. Journal of Sound and Vibration, Vol. 31, Issue 3, 1973, p. 309-314.

[16] Chen S. Vibration Theory of Structures with Random Parameters. Jilin Science and Technology Press, Changchun, 1990.

[17] Gere J., Goodno B. Mechanics of Materials (8 Edition). CENGAGE Learning Custom Publishing, Boston, 2013.

[18] Zhang Y., He X., Liu Q., Wen B. Robust reliability design of banjo flange with arbitrary distribution parameters. Journal of Pressure Vessel Technology - Transactions of the ASME, Vol. 27, Issue 4, 2005, p. 408-413.

[19] Roberts J. Structural fatigue under non-stationary random loading. Proceedings of the Institution of Mechanical Engineers, Part C, Journal of Mechanical Engineering Science, Vol. 8, Issue 4, 1966, p. 392-405.

[20] Rice S. Mathematical analysis of random noise. Bell System Technical Journal, Vol. 23, Issue 3, 1945, p. 282-332. 

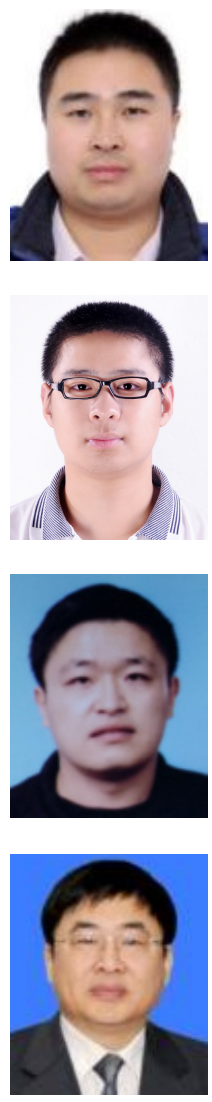

Zhang Yimin received Ph.D. degree in College of Mechanical Science and Engineering, Jilin University, Changchun, China, in 1995. Now he works at School of Mechanical Engineering and Automation, Northeastern University, Shenyang, China. His current research interests include dynamics and reliability engineering.

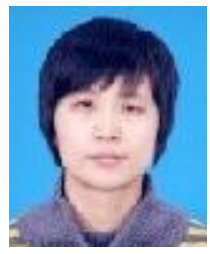

Li Changyou received Ph.D. degree in School of Astronautics, Harbin Institute of Technology, Harbin, China, in 2009. Now he is works at School of Mechanical Engineering and Automation, Northeastern University, Shenyang, China. His current research interests include dynamics and fault diagnosis

Wang Xuchu received Bachelor degree in College of Mechanical Engineering, Qingdao University of Technology, Qingdao, China, in 2014. Now he is currently a master candidate. His current research interests include dynamics and fault diagnosis.

Wang Wei received Master degree in School of Mechanical Engineering and Automation, Northeastern University, Shenyang, China, in 2015. Now he is currently a Ph.D. candidate. His current research interests include dynamics and fault diagnosis.

Guo Song received Ph.D. degree in College of Computer Science and Technology, Harbin Engineering University, Harbin, China, in 2011. Now he works at Information and Control Engineering Faculty, Shenyang Jianzhu University, Shenyang, China. His current research interests include image processing and fault diagnosis. 